\title{
Die Zusammenarbeit von Lehrkräften, Hochschule und außerschulischen Bildungsakteuren - kollaborative Materialentwicklung unter der Perspektive BNE
}

\begin{abstract}
Zusammenfassung
Bei der Integration von Bildung für nachhaltige Entwicklung in Schule und Unterricht fällt Lehrkräften als Multiplikatorinnen und Multiplikatoren eine Schlüsselrolle zu, jedoch fehlt es vielfach an Angeboten, um Lehrkräfte bei dieser Aufgabe zu unterstützen. Aus- und Weiterbildungsformate, in denen gezielt die Entwicklung notwendiger Kompetenzen von Lehrkräften verfolgt wird, rücken in diesem Zusammenhang vermehrt in den Fokus wissenschaftlicher Forschungs- und Entwicklungsarbeiten. Einen vielversprechenden Ansatz bieten langfristig angelegte Partnerschaften zwischen Schulen und außerschulischen Bildungsorten, in denen gemeinsam und lösungsorientiert an spezifischen nachhaltigkeitsrelevanten Frage- und Problemstellungen im Kontext Schule und Unterricht gearbeitet wird. Inwiefern diese Form der Zusammenarbeit dazu beitragen kann, einen wechselseitigen Wissenstransfer zu ermöglichen und so die Integration von BNE in die Unterrichtspraxis zu unterstützen, wird anhand der folgenden Fallstudie dargestellt. Hierfür wurde ein spezifisches transdisziplinäres Setting untersucht, in dessen Rahmen schulische und außerschulische Partnerinnen und Partner gemeinsam mit Forschenden Material unter der Perspektive BNE entwickeln.
\end{abstract}

Schlüsselworte: Bildung für nachhaltige Entwicklung, Community of Practice, Lehrkräftebildung

\footnotetext{
Abstract

Teachers play a key role as multipliers when it comes to integrating Education for Sustainable Development into schools. What is often lacking, however, is adequate support structures. In this context, initial and in-service training formats, which focus specifically on developing the necessary competencies of teachers, have become an increasing interest of scientific research and development work. One promising approach is longterm partnerships between schools and extracurricular venues, in which joint, solution-oriented collaboration on specific sustainability-related questions and problems in the context of school and teaching is fostered. The following case study illus-
}

trates to what extent this form of collaboration can contribute to a mutual transfer of knowledge and thus support the integration of ESD into teaching practice. For this purpose, a specific transdisciplinary setting was analyzed in which teachers and extracurricular partners develop material together with researchers under the perspective of ESD.

Keywords: education for sustainable development, community of practice, teacher education

\section{Einleitung}

Mit der Agenda 2030 verabschiedete die UN 17 Nachhaltigkeitsziele (Sustainable Development Goals), von denen eines explizit den Bildungssektor adressiert (Ziel 4: „Inklusive, gleichberechtigte und hochwertige Bildung gewährleisten und Möglichkeiten lebenslangen Lernen für alle fördern“) (DESA, 2015). Bildung für nachhaltige Entwicklung (BNE) als innovatives Bildungskonzept ermöglicht und unterstützt die Entwicklung zukunftsfähiger Kompetenzen zum Umgang mit Nachhaltigkeitsherausforderungen wie Klimawandel, Migration oder Gerechtigkeit (Bormann, 2013). Für das Erreichen einer nachhaltigen Entwicklung wird Bildung somit Ziel und Schlüssel zugleich. Doch obwohl die Notwendigkeit der Implementation von BNE in die schulische Praxis vielfach explizit formuliert wird und politische Forderungen und Maßgaben bestehen (u.a. UNESCO, 2014; KMK, 2017; Niedersächsisches Kultusministerium, 2017), ist BNE bis heute nicht strukturell und flächendeckend in Schule und Unterricht integriert (Brock, 2018). Lehrkräfte, denen als Multiplikatorinnen und Multiplikatoren eine wichtige Schlüsselrolle bei der Integration von BNE zufällt (UNESCO, 2014), beklagen häufig mangelnde Unterstützung bei der Herausforderung, Innovationen wie BNE umzusetzen (Gräsel, 2011). Die Befähigung von Lehrkräften, Unterricht aktiv unter BNE-Perspektive durch ein Set an dafür notwendigen Kompetenzen zu gestalten, gewinnt in diesem Kontext zunehmend an Bedeutung (Barth, 2015). In den Fokus wissenschaftlicher Forschungs- und Ent- 
wicklungsarbeiten rücken damit Aus- und Weiterbildungsformate, in denen Lehrkräfte gezielt die Möglichkeit erhalten, entsprechende Kompetenzen zu erwerben (u.a. Brandt et al., 2019; Künzli David, 2007; Redman et al., 2018). Kooperationskompetenz, zu der auch die Fähigkeit zur Kooperation mit externen Partnern zählt, ist laut einer Studie von Hellberg-Rode \& Schrüfer (2016) eine der elementaren Kompetenzen für eine erfolgreiche Umsetzung von BNE im schulischen Unterricht. Die Öffnung von Schule gegenüber Kooperationen mit außerschulischen Partnern birgt vor diesem Hintergrund großes Potenzial für die Implementation von BNE, da außerschulische Bildungsorte neben einem weitreichenden Repertoire an Bildungsangeboten für Schulen vielfach über ein vertieftes Konzeptwissen zu BNE verfügen (Reinke \& Hemmer, 2017). Gezielt langfristig angelegte Partnerschaften, die über das häufig übliche $\mathrm{Maß}$ an reiner „Buchung“ der außerschulischen Angebote hinausgehen, und in denen gemeinsam und lösungsorientiert an spezifischen nachhaltigkeitsrelevanten Frage- und Problemstellungen im Kontext Schule und Unterricht gearbeitet wird, können einen Wissenstransfer in beide Richtungen ermöglichen und somit die strukturelle Verankerung von $\mathrm{BNE}$ vorantreiben.

\section{Theoretischer Hintergrund}

Rund die Hälfte der Projekte und Maßnahmen, die im Rahmen der UN-Dekade Bildung für nachhaltige Entwicklung von 2005 bis 2015 offiziell ausgezeichnet wurden, entfällt auf den Bildungsbereich der außerschulischen Bildung und Weiterbildung (DUK, 2015). Der Einbezug außerschulischer Bildungsangebote in den regulären schulischen Unterricht schafft Gestaltungsräume, in denen Schülerinnen und Schülern eine Auseinandersetzung mit Nachhaltigkeitsfragestellungen und der Aufbau notwendiger Kompetenzen für die aktive Gestaltung nachhaltiger Entwicklungsprozesse ohne Leistungsanforderungen ermöglicht wird (Stoltenberg, 2013). Schulen und Lehrpersonen nehmen das umfangreiche Angebot außerschulischer Bildungsorte vielfach in Anspruch, da dieses gemeinhin als Erweiterung des schulischen Bildungsauftrags angesehen wird (Groß, 2011). Der Erfolg außerschulischen Lernens hängt dabei nach Thomas (2009) entscheidend von der Vor- und Nachbereitung der außerschulischen Lernerfahrung im Unterricht ab, jedoch zeigen Ergebnisse wissenschaftlicher Studien, dass dies bisher zu selten gelingt (u.a. Klaes, 2008a; Anderson \& Zhang, 2003). Als Voraussetzungen für eine erfolgreiche Integration von außerschulischen Bildungsangeboten in die schulische Unterrichtspraxis gelten laut Klaes (2008b) gegenseitiges Verständnis für die unterschiedlichen Bedarfe, Voraussetzungen und Möglichkeiten sowie ein gezielter Austausch des jeweiligen Professionswissens über die Grenzen der Institutionen hinweg. Eine zielgerichtete Kollaboration, die institutionenübergreifendes wechselseitiges Lernen mit- und voneinander ermöglicht und die Integration der vielfältigen Wissensbestände fördert, erscheint somit als notwendige Voraussetzung für eine umfassende Implementierung von BNE in das deutsche Bildungssystem und wird von BNE-erfahrenen Lehrkräften als erforderlich für eine erfolgreiche Umsetzung von BNE im Unterricht angesehen (Hellberg-Rode \& Schrüfer, 2016).
Transdisziplinäre Forschung, die in der Nachhaltigkeitswissenschaft Tradition hat, ermöglicht eine gemeinsame Bearbeitung gesellschaftlicher Herausforderungen und wissenschaftlicher Fragestellungen durch relevante Akteurinnen und Akteure aus Wissenschaft und Praxis (Rieckmann, 2015) und bietet hierduch vielfältige Anknüpfungspunkte, um Raum für ein solches institutionenübergreifendes wechselseitiges Lernen zu schaffen. Durch die Integration unterschiedlicher Wissensbestände können gemeinsame Problemlösungen entwickelt werden und zugleich Erkenntnisse von wissenschaftlicher Relevanz gewonnen werden (Lang et al., 2012). Anknüpfungspunkte für die konkrete Gestaltung innovativer Orte für transdisziplinäre Zusammenarbeit und die Ermöglichung wechselseitiger Lernprozesse bietet das Konzept der Communities of Practice (CoP), die nach Lave and Wenger (1991) Gemeinschaften sind, deren Mitglieder bestimmte Ziele teilen und Interessen verfolgen, um gemeinsam Wissen zu generieren und sich sowohl persönlich als auch professionell weiterzuentwickeln. CoPs leben von der grundsätzlich freiwilligen Teilnahme ihrer Mitglieder, Form und Grad der Teilhabe sind dabei jederzeit dynamisch und nicht von außen plan- oder gar steuerbar (Bliss et al., 2006). Die Mitglieder organisieren sich selbst und stehen über einen längeren Zeitraum hinweg in stetiger Interaktion miteinander (Wenger et al., 2002; Zboralski, 2007). Somit schaffen sie Räume, um nützliches Wissen für die Organisationen zu entwickeln, erhalten und zu teilen, denen die Mitglieder zugehörig sind (Agrifoglio, 2015). Eine präzisere Beschreibung der CoP schaffen Wenger et al. (2002) mit der Definition der drei Merkmale (1) Domain, (2) Community und (3) Practice als zentrale Voraussetzungen einer CoP. Das Merkmal Domain steht für den Wissensbereich, um den herum sich eine Gruppe bildet und definiert bestimmte Themen, mit denen die Mitglieder sich auseinandersetzen wollen. Sie ist Ausgangspunkt für die Fragestellungen und unterstützt den gemeinsamen Lernprozess durch das Schaffen einer gemeinsamen Identität und Zugehörigkeit, die weniger durch eine geteilte Aufgabe als vielmehr durch den Wissensbereich entsteht, der gemeinsam entwickelt werden soll (Agrifoglio, 2015; Wenger et al., 2002; Wenger, 2004). Das Merkmal Community steht für die sozialen Strukturen, die das Lernen durch Interaktion und die Beziehungen der CoP-Mitglieder untereinander fördern, da die Zusammenarbeit auf den zwischenmenschlichen Beziehungen fußt (Agrifoglio, 2015). Soziale Beziehungen begünstigen wechselseitiges Lernen, indem Diskussionen im Rahmen der Domain und die Entwicklung von Ideen ermöglicht werden und Engagement gefördert wird (ebd.). Regelmäßige Treffen und die Schaffung eines gemeinsamen Verständnisses der gemeinsamen Domain sind hierfür eine zentrale Voraussetzung (Wenger et al., 2002). Das dritte Merkmal Practice beschreibt die tatsächliche Praxis der CoP und bezieht sich auf das spezifische Wissen ihrer Mitglieder, das diese einbringen, gemeinsam entwickeln und untereinander teilen (Agrifoglio, 2015), um in ihrer Domain erfolgreich zu sein (Wenger et al., 2002). Die Entwicklung eines geteilten Repertoires an Ressourcen wie z.B. gemeinsame Routinen, Sichtweisen oder Vokabular unterstützt dabei die Wissensgenese (ebd.).

Inwiefern die Arbeit in CoPs zu Nachhaltigkeitsherausforderungen tatsächlich dazu beitragen kann, schulische und außerschulische Bildung enger miteinander zu verknüpfen und 
wechselseitige Lernprozesse zu initiieren, die die Integration von BNE in die Unterrichtspraxis unterstützen, wird anhand der folgenden Fallstudie dargestellt. Hierfür wird zunächst ein Beispiel eines spezifischen transdisziplinären Settings beschrieben und anschließend anhand eines konkreten Projekts zur kollaborativen Entwicklung von Material ausgeführt, wie Lernen in diesem Rahmen stattgefunden hat. Das empirische Design beschreibt anschließend die Gewinnung qualitativer Daten zur Beantwortung der Frage nach wechselseitigen Lernprozessen im Rahmen der Zusammenarbeit rund um die Materialentwicklung sowie möglichen Unterstützungsfaktoren. Die Ergebnisse werden anhand einiger Schlaglichter skizziert und abschließend diskutiert.

\section{Fallstudie „Kollaborative Materialentwick- lung im Entwicklungsteam Sachunter- richt"}

Das Setting: Entwicklungsteam Sachunterricht

Zur Ermöglichung von Räumen transdisziplinärer Zusammenarbeit wurde im Rahmen des Forschungs- und Entwicklungsprojekts „ZZL-Netzwerk“" an der Leuphana Universität Lüneburg das sog. Entwicklungsteam Sachunterricht gegründet, in dem Forschende der Leuphana mit Sachunterrichtslehrkräften sowie Vertreterinnen und Vertretern außerschulischer Bildungsorte zusammenarbeiten. Innerhalb des Entwicklungsteams (ET) werden Fragestellungen zu Herausforderungen schulischer Praxis in Zusammenhang mit BNE institutionenund phasenübergreifend generiert und aus gemeinsam identifizierten konkreten Bedarfen der Praxispartnerinnen und -partner werden spezifische Aufgabenstellungen abgeleitet. Diese werden einerseits in studentischen Projektseminaren sowie andererseits im ET selbst bearbeitet. Die dadurch generierten Outputs in Form von innovativen Konzepten und Unterrichtsmaterialien können nach einer anschließenden Erprobungsphase durch die Praktikerinnen und Praktiker des ETs an den beteiligten Schulen und zum Teil an den außerschulischen Bildungsorten eingesetzt werden und sollen somit Verstetigung in der Praxis finden (s. Abb. 1).

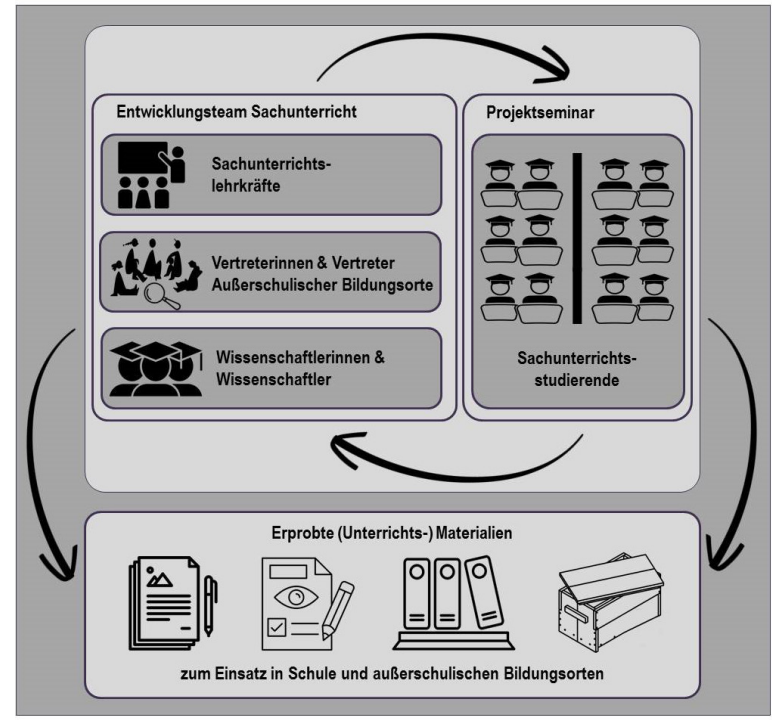

Abb. 1: Transdisziplinäres Entwicklungsteam Sachunterricht, Quelle: eigene Darstellung mit Icons von thenounproject.com

\section{Begleitforschung: Empirisches Design}

Den Ausgangspunkt der Kooperation im ET bildet die gemeinsame (1) Identifikation schulpraktischer Herausforderungen und Problemstellungen (s. Abbildung 2), der die (2) Reflexion und Analyse der eigenen Unterrichtspraxis unter Berücksichtigung der eingesetzten Materialien zu Grunde liegt. Im dritten Schritt werden (3) spezifische Bedarfe identifiziert und daraus konkrete Aufgabenstellungen formuliert. Die Aufgabenstellungen fokussieren die Entwicklung praxisfähiger Outputs in Form von Konzepten und Unterrichtsmaterialien unter der Perspektive BNE, die jeweils (4.1) im ET selbst oder (4.2b) im Seminar von Sachunterrichtsstudierenden des 4. Semesters im Bachelorstudiengang Lehren und Lernen erarbeitet werden (Bürgener \& Barth, 2018). Die Bearbeitung im ET (4.1) erfolgt unter Einbezug der Expertise aller Beteiligten. Nach der ersten Fertigstellung des Materials (durch die Studierenden

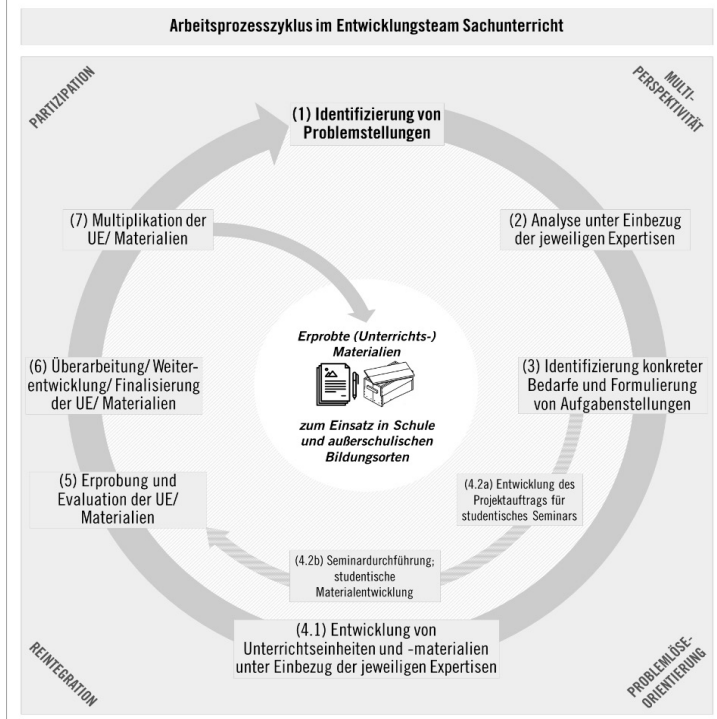

Abb. 2: Arbeitsprozesszyklus, Materialentwicklung im Entwicklungsteam Sachunterricht, Quelle: orientiert an Waschewski \& Weinhold (2020) 
bzw. das ET) wird dieses von den Praktikerinnen und Praktikern im eigenen Unterricht (5) erprobt und anschließend gemeinsam evaluiert. Auf die abschließende (6) Überarbeitung oder ggf. Weiterentwicklung und Finalisierung folgt die (7) Multiplikation des Materials in die beteiligten Schulen und außerschulischen Bildungsorte sowie das Studierendenseminar. Nach erfolgreichem Abschluss eines Projekts beginnt der Arbeitsprozess von vorn.

Die einzelnen Phasen sind dabei nicht immer eindeutig voneinander abzugrenzen und gehen teilweise dynamisch ineinander über. Der gesamte Arbeitsprozess im ET ist charakterisiert durch Problemlöseorientierung, Multiperspektivität, Partizipation sowie (Re-)Integration und orientiert sich damit an den vier Gestaltungsprinzipien transdisziplinärer Forschungs- und Entwicklungsarbeit (Straub \& Dollereder, 2019). Die Forschenden gestalten punktuell bedarfsorientierte Professionalisierungsangebote in Form von Kurzinputs oder spezifisch aufbereiteten Rückmeldungen zu den Arbeitsergebnissen. Zudem werden die Entwicklungsteammitglieder selbst zu Forschungssubjekten, die mittels qualitativer Forschungsmethoden Einblicke in ihre Lernprozesse geben und somit wissenschaftliche Erkenntnisse hinsichtlich der Expertiseentwicklung in Hinblick auf BNE liefern.

Das hier vorgestellte Fallbeispiel fokussiert auf das erste kollaborative Entwicklungsprojekt, das im ET Sachunterricht durchgeführt wurde. Im Mittelpunkt der Untersuchung steht die kollaborative Materialentwicklung zum Thema Umgang mit Ressourcen am Beispiel Papier durch das ET, eine parallele Bearbeitung derselben Aufgabenstellung im studentischen Seminar fand nicht statt. Die thematische Schwerpunktsetzung wurde von allen Beteiligten als besonders relevant angesehen, da eine Legitimation des Themas durch das Curriculum gegeben war, bestehende Materialien jedoch überwiegend als wenig anschlussfähig an den eigenen Unterricht oder unzureichend insbesondere unter der Perspektive BNE angesehen wurden.

Für die Gewährleistung der Anschlussfähigkeit an den verschiedenen Schulen und in unterschiedlichen Klassenstufen wurde als gemeinsame Herausforderung die Aufgabe for- muliert, ein generisches Modell einer in sich geschlossenen Unterrichtseinheit zum Thema Umgang mit Ressourcen am Beispiel Papier jeweils für die Jahrgangsstufen 1 und 2 sowie 3 und $4 \mathrm{zu}$ konzipieren und dieses anschließend in den einzelnen Schulen zu erproben und zu evaluieren (s. Abb. 3 für die einzelnen Schritte des Materialentwicklungsprozesses).

Das Projekt: Kollaborative Materialentwicklung

Die Begleitforschung fokussiert auf die im Fallbeispiel beschriebene transdisziplinäre Kollaboration und deren mögliches Potenzial als Treiber für wechselseitige Lernprozess im Kontext einer BNE:

- Inwiefern findet wechselseitiges Lernen zu BNE im Sinne einer CoP statt?

- Durch welche Faktoren werden die wechselseitigen Lernprozesse unterstützt?

Zu diesem Zweck wurden unterschiedliche qualitative Daten über den gesamten Entwicklungszeitraum vom Auftakttreffen im Dezember 2016 bis zur Erprobung der Einheit im April 2018 erhoben (s. Tabelle 1). Bei allen Treffen des ETs wurden teilnehmende Beobachtungen durchgeführt, Protokolle der Treffen angefertigt sowie Feldnotizen erstellt. Etwa zur Hälfte des Entwicklungsprozesses wurden leitfadenstützte Interviews mit vier der beteiligten Lehrkräfte sowie der Angehörigen des außerschulischen Bildungsortes geführt. Mit der fünften Lehrkraft wurde nach der Erprobung des Materials ein weiteres leitfadengestütztes Interview geführt.

Nach dem Abschluss der Entwicklungsarbeiten und vor der Pilotierung des Materials wurde zudem in einer Sitzung ein Audiomitschnitt angefertigt, in der die bisherige Zusammenarbeit gemeinsam mit allen Beteiligten reflektiert wurde. Die Interviews und der Audiomitschnitt wurden transkribiert und das gesamte schriftliche Material basierend auf der qualitativen Inhaltsanalyse nach Mayring (2015) ausgewertet. Die Kategorien wurden sowohl induktiv als auch deduktiv gebildet und in mehreren FeedbackSchleifen überarbeitet sowie deren Reliabilität überprüft.

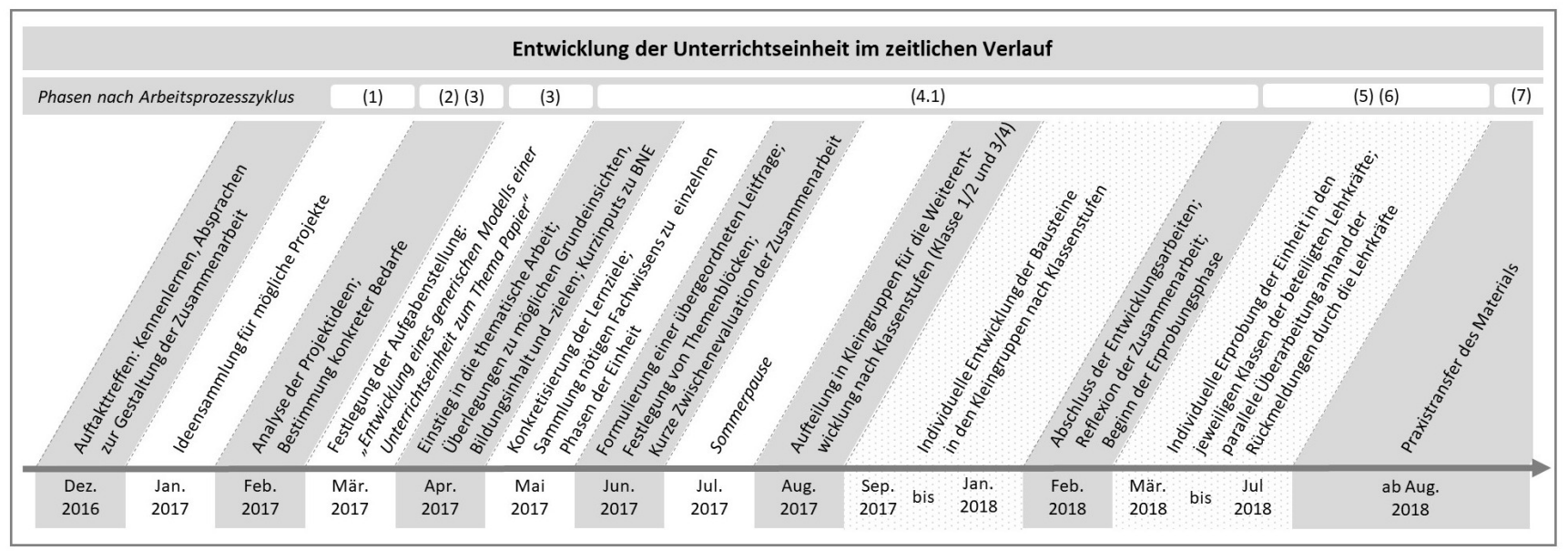

Abb. 3: Entwicklungsprozess der Unterrichtseinheit im zeitlichen Verlauf, Quelle: eigene Darstellung 


\begin{tabular}{|c|c|}
\hline $\begin{array}{l}\text { Leitfadengestützte } \\
\text { Interviews }\end{array}$ & $\begin{array}{l}\text { Reflexion der bisherigen } \\
\text { Zusammenarbeit, Fokus auf } \\
\text { wechselseitiges Lernen zu BNE } \\
\mathrm{N}=5 \text { im Juni } 2017 \text { (etwa zur Halbzeit } \\
\text { des Entwicklungsprozesses); } \\
\mathrm{N}=1 \text { im April } 2018 \text { (nach erstmaliger } \\
\text { Erprobung des Materials) } \\
\text { 28-56 min. }\end{array}$ \\
\hline $\begin{array}{l}\text { Audiomittschnitt } \\
\text { des } \\
\text { Entwicklungs- } \\
\text { teamtreffen }\end{array}$ & $\begin{array}{l}\text { Gemeinsame Reflexion der } \\
\text { Zusammenarbeit } \\
\mathrm{N}=1 \text { (Teilnehmende: } \mathrm{n}=7 \text { ) im Februar } \\
2018 \text { (nach Entwicklung und vor } \\
\text { Erprobung des Materials) } \\
120 \text { min. }\end{array}$ \\
\hline $\begin{array}{l}\text { Teilnehmende Be- } \\
\text { obachtung in den } \\
\text { Entwicklungs- } \\
\text { team- } \\
\text { treffen }\end{array}$ & $\begin{array}{l}\text { Schritte der kollaborativen Material- } \\
\text { entwicklung } \\
\mathrm{N}=11 \text { Protokolle plus Feldnotizen } \\
\text { (über den gesamten Verlauf des Ent- } \\
\text { wicklungsprozesses von Dezember } \\
2016 \text { bis Februar 2018) }\end{array}$ \\
\hline
\end{tabular}

Tab. 1: Datenerhebungen während des Entwicklungsprozesses, Quelle: eigene Darstellung

Über den gesamten Verlauf des Entwicklungsprozesses von etwa eineinhalb Jahren waren neben zwei Forschenden kontinuierlich fünf Sachunterrichtslehrkräfte von vier verschiedenen Grundschulen aus Stadt und Landkreis Lüneburg sowie eine Angehörige eines außerschulischen Bildungsortes aus Lüneburg beteiligt. Weitere Mitglieder schieden im Verlauf des Prozesses aus oder kamen später dazu und sind daher nicht Teil dieser Studie.

\section{Einblicke in die Ergebnisse}

Qualität der Zusammenarbeit

Die Mitglieder des ETs eint zunächst das übergeordnete Interesse an der Frage, wie Unterricht konkret unter der Perspektive BNE gestaltet werden kann. Sie teilen eine große Offenheit gegenüber Perspektiven und Sichtweisen anderer und sind daran interessiert, die eigene Praxis durch einen disziplinen- und institutionenübergreifenden Austausch weiterzuentwickeln. Äußere Rahmenbedingungen wie z.B. die Regelmäßigkeit der Treffen, deren Vorstrukturierung durch die Forschenden, Ergebnisprotokolle nach jedem Treffen, konkrete Absprachen und transparente Zielvereinbarungen bewerten die Teilnehmenden als Unterstützungsfaktoren für die Zusammenarbeit: „Also die Vorstrukturierung und generell der Ablauf, den finde ich ganz gut. Auch dass es Hinterher das Protokoll gibt zeitnah und sehr ausfübrlich, so mit den ganzen Anhängen, das finde ich total gut." (IET_102).

Die bereits von Beginn an hohe Motivation der ETMitglieder wird zum Einstieg in die Projektarbeit durch den kollaborativen Aushandlungsprozess zur möglichen Schwerpunktsetzung des Projekts weiter gesteigert. Die Identifizierung konkreter Bedarfe der einzelnen Beteiligten und die gemeinsame Zuspitzung auf die Entwicklung der Unterrichtseinheit zum Thema Papier unter BNE-Perspektive, die anschlussfähig für alle Beteiligten ist, schafft eine persönliche Involviertheit mit hoher Relevanz für die eigene Praxis: „Und jetzt ist halt die Aufgabenstellung auch so ein bisschen so, dass jeder auch sein eigenes immer im Hinterkopf hat und mitreflektiert. Deswegen glaube ich, werden alle auch so ein bisschen stärker aktiviert. "(IET_101). Die Verlässlichkeit bei der Teilnahme an den Treffen und in Hinblick auf Absprachen sowie das Engagement und die aktive Beteiligung aller Mitglieder an Diskussionen rund um den Anlass der Zusammenarbeit ermöglichen den Aufbau persönlicher Beziehungen unter den Teilnehmenden und fördern die Bildung eines Vertrauensverhältnisses: „Also ich bin jemand der sehr straight arbeite, gerne und auch klare Absprachen hat. Und das finde ich, läuft total gut. Es wird ganz klar gesagt, dann und dann Termin und ich schicke Protokoll oder ich hänge die und die Sachen an. Und das ist verlässlich. Und das ist was, womit ich sehr gerne arbeite. Mit verlässlichen Partnern. Und das finde ich, klappt total gut. " (IET_103). Gegenseitige Wertschätzung, die Begegnung auf Augenhöhe und die Reflexion der eigenen Kompetenzen, die in den Entwicklungsprozess eingebracht werden können, führen zu einer konstruktiven und produktiven Arbeitsatmosphäre. „Ich fahr immer total gerne da hin. Ich finde es ist ein total wertschätzender, netter Umgang miteinander. [...] Also ich habe das Gefühl, das ist immer sachlich. Also, dass jede Meinung aufjeden Fall akzeptiert ist." (IET_106).

\section{Methodisch-didaktisch fokussierter Austausch}

Der intensive fachliche Austausch mit Kolleginnen und Kollegen und die dadurch gewonnenen Einblicke in die Herangehensweisen, Erfahrungen und Zugänge anderer führen zu einer Perspektiven- und Wissenserweiterung in Bezug auf die Umsetzungsmöglichkeiten von BNE im eigenen Unterricht. Außerschulische Partner bekommen durch die gemeinsame Unterrichtsplanung und den dadurch ermöglichten intensiven Austausch vertiefte Einblicke in didaktisch-methodische $\mathrm{Zu}$ gänge, die sie im normalen Arbeitsalltag nicht erhalten. „Also, ich kriege jetzt da schon noch mal mehr mit, wie die Themen eingeführt werden, wie man die Schüler beteiligt. Also zu diesem intensiven Austausch komme ich sonst gar nicht." (IET_101).

Das über die kollaborative Materialentwicklung eingebrachte Fachwissen und die stark handlungsorientierte Herangehensweise des beteiligten außerschulischen Bildungsortes liefern vielfältige Inspirationen und Anregungen für die Praxis der Lehrkräfte. „So dieses ganz Handlungsorientierte. Wir machen ein Spiel dazu, [...], da fehlt mir manchmal die Kreativität. Und das hat mich sehr befruchtet, wo ich dachte, ja echt noch mehr spielerisch gucken." (IET_106).

Auch weiterführendes Hintergrundwissen zu den Angeboten des außerschulischen Bildungsortes, das die Lehrkräfte durch den engen Austausch erhalten, eröffnet zusätzliches Potenzial für dessen Einsatz und es entwickelt sich ein Vertrauensverhältnis, von dem beide Seiten durch die Anbahnung bzw. Intensivierung langfristiger Kooperationen profitieren: „Und da ist es auch noch mal echt, finde ich, so ein Bonusfaktor, weil wir sonst dann in dem Beratungsgesprächen, also wir können praktisch auf das aufbauen, was im Entwicklungsteam schon passiert ist. " (IET_101). Die Setzung wissenschaftlicher Impulse durch die Forschenden stärkt zum einen die theoretische Fundierung des Materials, zum anderen führt die intensive Auseinandersetzung mit Literatur und Konzepten beruhend auf aktuellen wissenschaftlichen Erkenntnissen zu einem Wissenszuwachs in Bezug auf die Möglichkeiten der Integration von BNE in den Unter- 
richt seitens der Praktikerinnen und Praktiker und eröffnet ihnen neue Zugänge und Anschlussmöglichkeiten. „[...]so Material, wie diese Querblicke ${ }^{2}$. [...]. Ich fand immer diesen didaktischen Kommentar total gut dazu. [...] Dass es nämlich doch auch wirklich gar nicht so groß immer sein muss" (IET_106).

Inhaltliche Auseinandersetzungen $\mathrm{zu}$ Themen einer nachhaltigen Entwicklung finden zwar statt, eine dadurch erzeugte Perspektivenerweiterung wird von den ET-Mitgliedern jedoch nur sehr vereinzelt hervorgehoben: „Also ich fand halt, als er [außerschulischer Praxispartner] mit so einer ganz neuen Perspektive, da [...] wollte er eigentlich nicht, dass der Papierverbrauch dann so verteufelt wird und die ganze Wirtschaft, die da dran hängt sozusagen, weil er aus der Forstwirtschaft kommt. Und das war für mich so ein Aspekt, den ich jetzt als gar nicht so wichtig, sag ich jetzt mal ganz ehrlich, erachtet hab. [...] und dass man natürlich auch sagen muss, ja, es kann auch eher Landwirtschaft, Forstwirtschaft in diesem Fall, kann ja eben auch nachhaltig betrieben werden. [...]" (IET_105). Insgesamt ist festzuhalten, dass für die Praktikerinnen und Praktiker der Austausch und die Wissenserweiterung in Bezug auf methodisch-didaktische Fragen und Herausforderungen als besonders gewinnbringender Faktor der Zusammenarbeit angesehen wird.

\section{Potenzial für den Praxistransfer von BNE}

Die gemeinsame Entwicklungsarbeit in Verbindung mit der Auseinandersetzung über Möglichkeiten der praktischen Implementierung erzeugt einerseits ein vertieftes konzeptionelles Verständnis von BNE seitens der Lehrkräfte und führt andererseits zu einer Steigerung der Motivation, die erarbeiteten und kennengelernten Konzepte, Herangehensweisen und $\mathrm{Zu}-$ gänge (auch längerfristig) in den eigenen Unterricht integrieren. "Dass man das versucht in mehrere Unterrichtseinheiten immer wieder rein zu bringen. Damit die Kinder da auch nicht so auf einmal mit konfrontiert sind, sondern regelmäßig immer wieder diese Fragen sich stellen, wo wollen wir eigentlich hin?" (IET_104).

Die Diffusion des erarbeiteten Materials sowie des durch die Entwicklungsarbeit erzeugten Wissens in die Schulen gestaltet sich hingegen insgesamt schwierig und ist stark abhängig von der Offenheit des Kollegiums und der Schulleitung. Während einige ET-Mitglieder beispielsweise auf Dienstbesprechungen über Arbeit und Ergebnisse des ETs berichten, findet an anderen Schulen keinerlei Austausch statt. Es zeigt sich, dass vereinzelt über ET-Mitglieder, die Funktionsstellen innehaben (z.B. Fachkonferenzleitung) eine direkte Verankerung von BNE bspw. bei der Neugestaltung von Arbeitsplänen möglich werden kann: „Ein neuer Arbeitsplan ist ja auf Kompetenzen aufgebaut in erster Linie. Und vielleicht, also da sind wir gerade dabei, das zu schreiben und versuchen das auch zu berücksichtigen. Vielleicht kann man das da jetzt irgendwie dann besser berücksichtigen, noch weiter berücksichtigen" (IET_105). Die Weitergabe des erarbeiteten Materials findet hingegen überwiegend im persönlichen Kontakt mit engen Kolleginnen und Kollegen, weniger gezielt in das gesamte Kollegium statt. Über die Beratung und Fortbildung von Lehrkräften ergibt sich jedoch zusätzlich ein indirektes Transferpotential über den außerschulischen Partner: „[...] also das, was ich jetzt auch von den Lehrerinnen mitnehme, was ich aus den Querblicken mitnehme, das wird aufjeden Fall in die Beratungen einfließen an den Schulen." (IET_101)

\section{Diskussion und Ausblick}

Die Ergebnisse zeigen, dass ein wechselseitiges von- und miteinander Lernen zu BNE im vorgestellten Setting ermöglicht wird und Professionalisierungsprozesse sowohl seitens der Lehrkräfte als auch des außerschulischen Akteurs insbesondere auf didaktisch-methodischer Ebene angestoßen werden. Die Gestaltung der äußeren Rahmenbedingungen wirkt unterstützend auf die wechselseitigen Lernprozesse und auch die sich entwickelnde Qualität der Beziehungen zeigt sich in diesem Zusammenhang fördernd für die Ermöglichung der Professionalisierungsprozesse. Dadurch eröffnet diese Form der Zusammenarbeit die Möglichkeit einer engeren Verknüpfung von schulischer und außerschulischer Bildung. Ein Transfer von BNE in die schulische Praxis wird durch diesen Ansatz sowohl direkt (über die involvierten Lehrkräfte) als auch indirekt (über den außerschulischen Partner) ermöglicht, die Ergebnisse bestätigen jedoch die Annahme, dass dies insbesondere abhängig von Motivation und Engagement der Lehrkräfte (Trempler et al., 2013) und der Haltung von Kollegien und Schulleitungen ist (Stoltenberg, 2014). Es erscheint daher sinnvoll, gezielt Lehrkräfte auf Funktionsstellen und Schulleitungen mit in die Entwicklungsarbeit einzubeziehen und die Bildung von Tandems durch die Integration weiterer interessierter Lehrkräfte der beteiligten Schulen zu forcieren, um einen größeren Rückhalt in kritischen oder ablehnenden Kollegien zu erreichen.

Bereits durch die Freiwilligkeit der Teilnahme gilt für die Zusammenarbeit im ET eine positive „Vorauswahl“, indem sich auch hier die schon zuvor engagierten Praktikerinnen und Praktiker einbringen, die sich durch ein intrinsisch motiviertes Interesse an der Auseinandersetzung mit BNE-relevanten Fragestellungen und eine hohe Innovationsbereitschaft auszeichnen. Auch daran wird die Bedeutsamkeit der Motivation und des Engagements von Lehrkräften für den Transfer von BNE in die Praxis deutlich (u.a. Vare 2018; Bertschy et al., 2013; Trempler et al., 2013). Die Gestaltung von ansprechendem, inhaltlich überzeugendem und für verschiedene Lerngruppen anschlussfähigem Material könnte eine Möglichkeit sein, auch diejenigen Lehrkräfte zu erreichen, die BNE nach wie vor als Zusatzaufgabe sehen. Eine zusätzliche Steigerung der Reichweite und ein erhöhter Transfer in die Praxis ließe sich über die Veröffentlichung des Materials (z.B. über Open Educational Resources) erreichen und ist im Rahmen einer durch die Hochschule herausgegebenen Materialreihe geplant.

Es bleibt festzuhalten, dass der Transfer von BNE in Schule und Unterricht über den Weg der kollaborativen Materialentwicklung insbesondere abhängig von Motivation, Engagement und Veränderungswille der Praktikerinnen und Praktiker ist sowie einer intensiven Begleitung durch die Forschenden sowohl einerseits in Hinblick auf Schaffung der Rahmenbedingungen und der Setzung wissenschaftlicher Impulse sowie andererseits der kontinuierlichen wissenschaftlichen Begleitforschung bedarf. Die Fallstudie kann an dieser Stelle erste Hinweise für das Transferpotenzial des beschriebenen Settings liefern, inwiefern tatsächlich eine langfristige Wirkung auf die schulische Praxis ermöglicht wird, bleibt Anknüpfungspunkt für weitere Forschung. 


\section{Anmerkungen}

1 Gefördert durch die Qualitätsoffensive Lehrerbildung des BMBF, 1. Förderphase von 2016-2019.

2 Die Lehrmittelreihe „Querblicke“ basiert auf einem Verständnis von BNE als Vermittlung von spezifischen Kompetenzen nach Künzli and Bertschy (2008). Vgl hierzu auch https://www.querblicke.ch

\section{Literatur}

Agrifoglio, R. (2015). Knowledge preservation through community of practice: Theoretical issues and empirical evidence. SpringerBriefs in information systems. Cham: Springer. https://doi.org/10.1007/978-3-319-22234-9

Anderson, D. \& Zhang, Z. (2003). Teacher Perceptions of Field-Trip Planning and Implementation. Visitor Studies Today, 6(3), 6-11.

Barth, M. (2015). Implementing sustainability in higher education: Learning in an age of transformation. Routledge studies in sustainable development. London: Routledge. https://doi.org/10.4324/9780203488355

Bertschy, F., Künzli David, C. \& Lehmann, M. (2013). Teachers' Competencies for the Implementation of Educational Offers in the Field of Education for Sustainable Development. Sustainability, 5(12), S. 5067-5080. https://doi.org/10.3390/su512 5067

Bliss, F. R., Johanning, A., \& Schicke, H. (2006). Communities of Practice - Ein Zugang zu sozialer Wissensgenerierung. o.O: O.V.

Bormann, I. (2013). Bildung für nachhaltige Entwicklung als Praxis sozialer Innovation. In J. Rückert-John (Hrsg.), Research. Soziale Innovation und Nachhaltigkeit: Perspektiven sozialen Wandels (Vol. 25, S. 269-288). Wiesbaden: Springer VS. https://doi.org/10.1007/978-3-531-18974-1_14

Brandt, J.-O., Bürgener, L., Barth, M., \& Redman, A. (2019). Becoming a competent teacher in education for sustainable development. International Journal of Sustainability in Higher Education, 20(4), 630-653. https://doi.org/10.1108/IJSHE-10 $-2018-0183$

Brock, A. (2018). Verankerung von Bildung für nachhaltige Entwicklung im Bildungsbereich Schule. In A. Brock, G. d. Haan, N. Etzkorn, \& M. Singer-Brodowski (Hrsg.), Schriftenreihe „Ökologie und Erziehungswissenschaft" der Kommission Bildung für eine nachhaltige Entwicklung der DGfE. Wegmarken zur Transformation: Nationales Monitoring von Bildung für nachhaltige Entwicklung in Deutschland (S. 67115). Opladen u.a.: Barbara Budrich. https://doi.org/10.2307/j.ctvddzt7n.8

Bürgener, L. \& Barth, M. (2018). Sustainability competencies in teacher education: Making teacher education count in everyday school practice. Journal of Cleaner Production, 174, 821-826. https://doi.org/10.1016/j.jclepro.2017.10.263

DESA (2015). Sustainable Development Goals: 17 Goals to Transform our World. Zugriff am 15.06.2020 https://sustainabledevelopment.un.org/

DUK (2015). UN-Dekade mit Wirkung: 10 Jahre „Bildung für nachhaltige Entwicklung" in Deutschland. Bonn: o.V.

Gräsel, C. (2011). Die Kooperation von Forschung und Lehrer/innen bei der Realisierung didaktischer Innovationen. In W. Einsiedler (Hrsg.), Unterrichtsentwicklung und Didaktische Entwicklungsforschung (S. 88-101). Bad Heilbrunn: Klinkhardt.

Groß, J. (2011). Orte zum Lernen - Ein kritischer Blick auf außerschulische Lehr-Lernprozesse. In K. Messmer, R. von Niederhäusern, A. Rempfler, \& M. Wilhelm (Hrsg.), Außerschulische Lernorte - Beiträge zur Didaktik: Band 1. Ausserschulische Lernorte-Positionen aus Geographie, Geschichte und Naturwissenschaften. Wien, Berlin: LIT.

Hellberg-Rode, G. \& Schrüfer, G. (2016): Welche spezifischen professionellen Handlungskompetenzen benötigen Lehrkräfte für die Umsetzung von Bildung für Nachhaltige Entwicklung (BNE) - Ergebnisse einer explorativen Studie. Biologie Lehren und Lernen - Zeitschrift für Didaktik der Biologie, 20(1), 1-29.

Klaes, E. (2008a). Außerschulische Lernorte im naturwissenschaftlichen Unterricht: Die Perspektive der Lehrkraft. Studien zum Physik-und Chemielernen. Berlin: Logos.

Klaes, E. (2008b). Stand der Forschung zum Lehren und Lernen an außerschulischen Lernorten. In D. Höttecke (Hrsg.), Gesellschaft für Didaktik der Chemie und Physik (GDCP). Kompetenzen, Kompetenzmodelle, Kompetenzentwicklung - Empirische Forschung in den Fachdidaktiken. Jahrestagung der GDCP in Essen 2007 (S. 263-265). Münster: LIT.

KMK (2017). Zur Situation und zu Perspektiven der Bildung für nachhaltige Entwicklung: Bericht der Kultusministerkonferenz vom 17.03.2017. Zugriff am 16.07.2020 https://www.kmk.org/fileadmin/Dateien/veroeffentlichungen_beschluesse/2017 12017_03_17-Bericht-BNE-2017.pdf

Künzli, C., \& Bertschy, F. (2008). Didaktisches Konzept Bildung für eine nachhaltige Entwicklung: Arbeitspapier Nr. 1 aus dem Forschungsprojekt des Nationalfonds und der Lehrerinnen- und Lehrerbildung Bern: „Bildung für eine nachhaltige Entwicklung: Didaktische Konzeption und Umsetzung in die Schulpraxis". Bern: o.V.
Künzli David, C. (2007). Zukunft mitgestalten: Bildung für eine nachhaltige Entwicklung - didaktisches Konzept und Umsetzung in der Grundschule. PRISMA - Beiträge zur Erziehungswissenschaft aus historischer, psychologischer und soziologischer Perspektive. Bern: Haupt.

Lang, D. J., Wiek, A., Bergmann, M., Stauffacher, M., Martens, P., Moll, P., \& Thomas, C. J. (2012). Transdisciplinary research in sustainability science: practice, principles, and challenges. Sustainability Science, 7(1), 25-43. https://doi.org/10.100 7/s11625-011-0149-x

Lave, J., \& Wenger, E. (1991). Situated learning: Legitimate peripheral participation. Learning in doing. Cambridge: Cambridge Univ. Press. https://doi.org/10.1017/CB O9780511815355

Mayring, P. (2015). Qualitative Inhaltsanalyse: Grundlagen und Techniken (12., überarbeitete Auflage). Beltz Pädagogik. Weinheim: Beltz.

Niedersächsisches Kultusministerium (2017). Kerncurriculum für die Grundschule Schuljahrgänge 1-4: Sachunterricht. Hannover: o.V.

Redman, E., Wiek, A., \& Redman, A. (2018). Continuing Professional Development in Sustainability Education for K-12 Teachers: Principles, Programme, Applications, Outlook. Journal of Education for Sustainable Development, 12(1), 59-80. https://doi.org/10.1177/2455133318777182

Reinke, V., \& Hemmer, I. (2017). Bildung für nachhaltige Entwicklung - über welche Kompetenz verfügen Lehrkräfte und Akteur/-innen aus den außerschulischen Einrichtungen? Zeitschrift Zlb.Ku, 1(1), 38-43.

Rieckmann, M. (2015). Transdisziplinäre Forschung und Lehre als Brücke zwischen Zivilgesellschaft und Hochschulen. Zeitschrift Für Internationale Bildungsforschung und Entwicklungspolitik, 38(3), 4-10.

Stoltenberg, U. (2013). Sachunterricht für das 21. Jahrhundert: Weltorientierung durch Bildung für eine nachhaltige Entwicklung. In U. Stoltenberg (Hrsg.), Bildung für eine nachhaltige Entwicklung in Kindergarten und Grundschule: Bd. 1. Weltorientierung durch Bildung für eine nachhaltige Entwicklung. Theoretische Grundlagen und Praxis des Sachunterrichts in der Grundschule (S. 13-45). Bad Homburg, Waldkirchen: VAS, Südost-Verl.-Service.

Stoltenberg, U. (2014). Potenziale für Kinder und Gesellschaft. Frühkindliche Bildung als Bildung für eine nachhaltige Entwicklung. In Umweltdachverband (Hrsg.), Forum edition Jahrbuch - Bildung für nachhaltige Entwicklung. Krisen- und Transformationsszenarios: Frühkindpädagogik, Resilienz \& Weltaktionsprogramm (S. 47-57). Wien: o.V.

Thomas, B. (2009). Lernorte außerhalb der Schule. In K.-H. Arnold, U. Sandfuchs, \& J. Wiechmann (Hrsg.), Handbuch Unterricht (2. Auflage, S. 283-287). Bad Heilbrunn: Klinkhardt.

Trempler, K., Schellenbach-Zell, J. \& Gräsel, C. (2013). Der Einfluss der Motivation von Lehrpersonen auf den Transfer von Innovationen. In M. Rürup \& I. Bormann (Hrsg.), Educational governance: Innovationen im Bildungswesen: Analytische Zugänge und empirische Befunde (21), 329-347. https://doi.org/10.1007/9783-531-19701-2_14

UNESCO (2014). UNESCO-Roadmap zur Umsetzung des Weltaktionsprogramms "Bildung für nachhaltige Entwicklung". Bonn: Dt. UNESCO-Kommision.

Vare, P. (2018) A rounder sense of purpose: developing and assessing competences for educators of sustainable development. Form@re, 18(2), 164-173.

Waschewski, T. \& Weinhold, S. (2020). Kooperativ forschen und Unterricht entwickeln. Journal für LehrerInnenbildung, 20(2), 94-105.

Wenger, E. (2004). Knowledge management as a doughnut: Shaping your knowledge strategy through communities of practice. Ivey Business Journal, 68(1), 1-8.

Wenger, E., McDermott, R. \& Snyder, W. M. (2002). Cultivating communities of practice: A guide to managing knowledge. Boston, Mass.: Harvard Business School Press.

Zboralski, K. (2007). Wissensmanagement durch Communities of Practice. Strategisches Kompetenz-Management. Wiesbaden: DUV Deutscher Universitäts-Verlag.

\section{Lina Bürgener}

ist Doktorandin an der Leuphana Universität Lüneburg und forscht zur Entwicklung von Nachhaltigkeitskompetenzen in der Lehrkräftebildung. Besonderes Forschungsinteresse: Kompetenzerwerb von (angehenden) Lehrkräften durch neue Formen der Kollaboration zwischen Theorie und Praxis in innovativen Lehr-Lern-Formaten.

\section{Dr. Matthias Barth}

ist Professor für Sachunterricht und Bildung für nachhaltige Entwicklung an der Leuphana Universität Lüneburg. Forschungsschwerpunkt: Lernprozesse und nachhaltige Entwicklung, mit besonderem Fokus auf Kompetenzerwerb der Lernenden. 